\title{
Identification of a Single Nucleotide Change in the Hypoxanthine-Guanine Phosphoribosyltransferase Gene (HPRTYale) Responsible for Lesch-Nyhan Syndrome
}

\author{
Shin Fujimori, ${ }^{\star}$ Beverly L. Davidson, ${ }^{\ddagger}$ William N. Kelley, ${ }^{\star \neq 5}$ and Thomas D. Palella ${ }^{\star \xi}$ \\ Departments of ${ }^{*}$ Internal Medicine and ${ }^{\ddagger}$ Biological Chemistry, and ${ }^{\S}$ the Rackham Arthritis Research Unit, \\ University of Michigan Medical School, Ann Arbor, Michigan 48109
}

\begin{abstract}
Complete deficiency of hypoxanthine-guanine phosphoribosyltransferase (HPRT) causes the Lesch-Nyhan syndrome. Previous characterization of a mutant form of HPRT, HPRT $_{\text {Yale, }}$ from a subject with the Lesch-Nyhan syndrome revealed normal mRNA and protein concentrations, no residual catalytic activity, and cathodal migration upon PAGE. We have cloned and sequenced HPRT $_{\text {Yale }}$ CDNA. The nucleotide sequence of full-length HPRT $_{\text {Yale }}$ CDNA revealed a single nucleotide substitution compared with normal HPRT cDNA: $\mathbf{G} \rightarrow \mathbf{C}$ at nucleotide position 211. This transversion predicts substitution of arginine for glycine at amino acid position 71, explaining the cathodal migration of HPRT $_{\text {Yale }}$. Chou-Fasman secondary structure analysis predicts a change in the probability of $\beta$-turn formation in the region containing the mutation. Inclusion of the bulky arginine side chain in place of glycine probably disrupts protein folding as well. Cloning mutant forms of cDNA allows identification of specific mutations, provides insight into mutational mechanisms, and facilitates structure-function analysis of mutant proteins.
\end{abstract}

\section{Introduction}

The purine salvage enzyme hypoxanthine-guanine phosphoribosyltransferase (HPRT; ${ }^{1}$ EC 2.4.2.8) is encoded by an Xlinked gene in humans. Complete deficiency of HPRT causes the Lesch-Nyhan syndrome (1), while partial deficiency of this enzyme leads to purine overproduction and gout (2). Single amino acid substitutions have been identified in four HPRT variants by comparative mapping of tryptic peptides and sequencing of the aberrant peptides (3). However, most HPRTdeficient subjects have insufficient amounts of mutant protein for this type of analysis. Furthermore, the HPRT gene is very large $(44 \mathrm{~kb})$ and Southern blot analyses of genomic DNA from most HPRT-deficient subjects with HPRT cDNA probes are normal (4). On the other hand, the majority of HPRT-defi-

Address reprint requests to Dr. Thomas D. Palella, University of Michigan, Department of Internal Medicine, $1150 \mathrm{~W}$. Medical Center Drive, 5520 Medical Science Research Building I, Ann Arbor, MI 48109.

Received for publication 10 November 1987 and in revised form 27 July 1988.

1. Abbreviations used in this paper: HPRT, hypoxanthine-guanine phosphoribosyltransferase.

J. Clin. Invest.

(c) The American Society for Clinical Investigation, Inc.

$0021-9738 / 89 / 01 / 0011 / 03 \quad \$ 2.00$

Volume 83, January 1989, 11-13 cient subjects have normal quantities of HPRT mRNA (5). RNase mapping analysis can be used to detect mutations within mRNA in some mutant subjects (6). Allele-specific oligonucleotides in conjunction with RNase mapping allow deduction of certain nucleotide substitutions (7). However, this technique is limited to mutations for which the nucleotide substitution is already known. Therefore, in most cases where sufficient mRNA is present, mutant cDNA cloning currently is the method of choice for defining mutations at the HPRT locus. The amino acid substitution in a fifth variant has been deduced from the nucleotide sequence of a full-length cDNA cloned from a subject with partial HPRT deficiency (8). These previous studies are summarized in Table $I$.

In the present study, we have cloned and sequenced a fulllength HPRT cDNA from a patient with the Lesch-Nyhan syndrome. We have identified a single change in codon 71 (GGC $\rightarrow$ CGC), which predicts an amino acid substitution of arginine for glycine. This HPRT variant, which was previously termed HPRT $_{\text {Yale, }}$, is characterized by a normal intracellular concentration of enzyme protein, reduced catalytic activity, and cathodal migration in native PAGE (5). The altered charge of this mutant enzyme is consistent with the predicted substitution of a neutral amino acid (glycine) by a basic amino acid (arginine).

\section{Methods}

\section{Materials}

Lambda gt 11 arms and in vitro packaging extract were purchased from Vector Cloning Systems, San Diego, CA. M1 3 sequencing vectors and universal primer were obtained from Pharmacia Fine Chemicals, Piscataway, NJ. The 956-bp normal HPRT cDNA was kindly provided by C. Thomas Caskey of Baylor University, Houston, TX (9). HPRTspecific synthetic oligomers used in priming sequencing reactions were synthesized on a DNA synthesizer (model 380A; Applied Biosystems, Inc., Foster City, CA) by the Center for Molecular Genetics, University of Michigan. Avian myeloblastosis virus reverse transcriptase was obtained from Life Science, St. Petersburg, FL. All other enzymes were purchased from New England Biolabs, Beverly, MA. Radioactive nucleotides were obtained from New England Nuclear, Boston, MA. All other reagents were of the highest quality commercially available.

\section{Methods}

cDNA cloning and sequence analysis. EBV-transformed lymphoblast cell lines derived from patient $\mathrm{K}$. T. (HPRT $\mathrm{Yale}_{\text {ale }}$ ) were harvested from suspension cultures and lysed, and total RNA was extracted (10). Poly $\mathrm{A}^{+}$mRNA was purified by oligo-d(T) column chromatography (11). RNase mapping analysis of this mRNA was performed as described (6) using human HPRT riboprobes spanning the entire HPRT coding sequence. Both first and second strands of cDNA were synthesized with avian myeloblastosis virus reverse transcriptase according to the method of Polites and Marotti (12). The double-stranded cDNA was blunt-ended with T4 DNA polymerase and ligated to Eco RI linkers. 
Table I. Structural and Functional Abnormalities in Five HPRT Variants

\begin{tabular}{|c|c|c|c|c|c|c|c|c|}
\hline & \multirow[b]{2}{*}{$\begin{array}{l}\text { Specific } \\
\text { activity }\end{array}$} & \multirow[b]{2}{*}{$\begin{array}{l}\text { Immunoreactive } \\
\text { protein (CRM) }\end{array}$} & \multicolumn{2}{|c|}{ Michaelis constants } & \multirow[b]{2}{*}{ Isoelectric point } & \multirow[b]{2}{*}{$\begin{array}{l}\text { Amino acid } \\
\text { substitution }\end{array}$} & \multirow[b]{2}{*}{ Position } & \multirow[b]{2}{*}{ Reference } \\
\hline & & & Hypoxanthine & PRPP & & & & \\
\hline & $\%$ control & $\%$ control & & & & & & \\
\hline HPRT $_{\text {Toronto }}$ & 33 & 52 & Normal & Normal & Acidic & Arg $\rightarrow$ Gly & $(50)$ & $(3,6)$ \\
\hline HPRT $_{\text {Munich }}$ & 3 & 79 & Increased 75 -fold & Normal & Basic & Ser $\rightarrow$ Arg & (103) & $(3,6)$ \\
\hline HPRT $_{\text {London }}$ & 59 & 35 & Increased 5-fold & Normal & Normal & Ser $\rightarrow$ Leu & (109) & $(3,6)$ \\
\hline HPRT $_{\text {Ann Arbor }}$ & 10 & 11 & Increased 10 -fold & Increased 2-fold & Acidic & Ile $\rightarrow$ Met & $(132)^{*}$ & $(6,8)$ \\
\hline HPRT $_{\text {Kinston }}$ & $<0.7$ & 72 & Increased & Increased & Basic & Asp $\rightarrow$ Asn & (193) & $(3,6)$ \\
\hline
\end{tabular}

Arg, arginine; Gly, glycine; Ser, serine; Leu, leucine; Ile, isoleucine; Met, methionine; Asp, aspartic acid; Asn, asparagine. ${ }^{*}$ The initial methio-

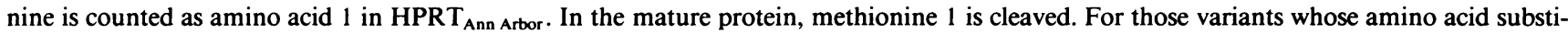
tution was determined by amino acid sequencing, alanine was counted as amino acid 1 .

After digestion with Eco RI the cDNA was purified by Sepharose CL4B column chromatography and ligated to Eco RI-digested lambda gt 11 arms. The resultant cDNA library of $\sim 260,000$ phage clones was screened by using a ${ }^{32}$ P-labeled normal HPRT cDNA (9) according to the method of Benton and Davis (13). For sequence analysis, the inserts of positive clones were subcloned in M13 vectors. DNA sequencing was carried out by the dideoxynucleotide chain termination method $(14,15)$.

$\beta$-turn prediction analysis. The relative probability that a tetrapeptide will form a $\beta$-turn (Pt) was calculated from $P t=f_{i} \times f_{i+1} \times f_{i+2}$ $\times f_{i+3}$ according to the protein conformational prediction method of Chou and Fasman (16). The $f_{i}, f_{i+1}, f_{i+2}$, and $f_{i+3}$ are bend frequencies in the four positions of the $\beta$-turn. Bend frequencies are based on a statistical analysis of 29 proteins (16). As a reasonable cut-off value in predicting $\beta$-turn, $\mathrm{Pt}=1.0 \times 10^{-4}$ was chosen (17).

\section{Results}

Two positive clones were found on screening $\sim 260,000$ recombinant phage plaques as described in Methods. The sizes of the cDNA inserts from these clones were determined to be 1.4 and $1.0 \mathrm{~kb}$ by Southern blot analysis after Eco RI digestion of phage DNA. Both cDNA inserts were subcloned to M13mp19 and sequenced using synthetic oligomers and the universal primer. The 1.4-kb cDNA, designated $\mathrm{pHKT}_{1}$, was digested with Hae III and then subcloned in M13mp19 for determination of $3^{\prime}$ noncoding sequence. Since RNase mapping analyses of HPRT Yale $_{\text {mRNA using normal HPRT ribo- }}$ probes spanning the entire coding sequence were normal (data not shown), $\mathrm{pHKT}_{1}$ was sequenced in its entirety, and the coding sequence of $\mathrm{pHKT}_{1}$ was completed in both directions. It shows a single nucleotide change $(G \rightarrow C)$ at nucleotide position 211 (Fig. 1). This $\mathrm{G}$ to $\mathrm{C}$ change predicts an amino acid substitution from glycine (GGC) to arginine (CGC) in codon 71 (Fig. 2). Sequencing of the 1.0-kb cDNA clone, which is 138 nucleotides short of the entire coding sequence, also revealed only the same nucleotide change. This nucleotide
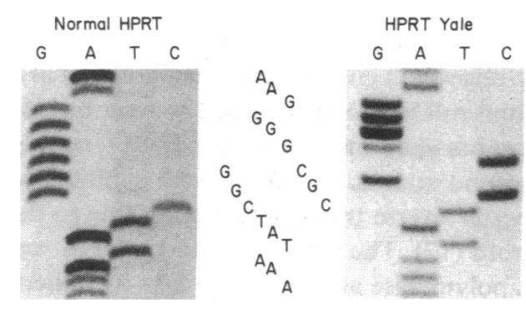

Figure 1. Comparison of the nucleotide sequences of normal HPRT and HPRT Yale $_{\text {. }}$ Sequences from base positions 205-219 are shown with the $G$ to $C$ transversion identified at position 211 . change does not alter any recognition site for a restriction enzyme within the HPRT gene.

The probabilities of $\beta$-turn occurrence were calculated for the 11 tetrapeptide combinations of the 14 amino acid residues in position 64-77 of normal HPRT (Fig. 3). The Pt values at residues $68-71$ and $69-72$ were $2.0 \times 10^{-4}$ and $0.6 \times 10^{-4}$, respectively, and the $\mathrm{Pt}$ value at residues $70-73$ increased from $0.9 \times 10^{-4}$ to $1.2 \times 10^{-4}$ (Fig. 3 ).

\section{Discussion}

We have defined the mutation of a mutant HPRT (HPRT Yale $)$ isolated from a patient with the Lesch-Nyhan syndrome. The predominant abnormality in most patients with the LeschNyhan syndrome is reduced concentration of enzyme protein rather than a defect in enzyme function (5). HPRT $_{\text {Yale }}$ belongs to the latter class of mutants. In previous studies HPRT Yale $_{\text {was }}$ shown to have an almost normal concentration of enzyme protein, no detectable catalytic activity, and a cathodal migration in native PAGE (5). The mutation in HPRT $_{\text {Yale }}$ should therefore affect catalytic function rather than protein synthesis or degradation.

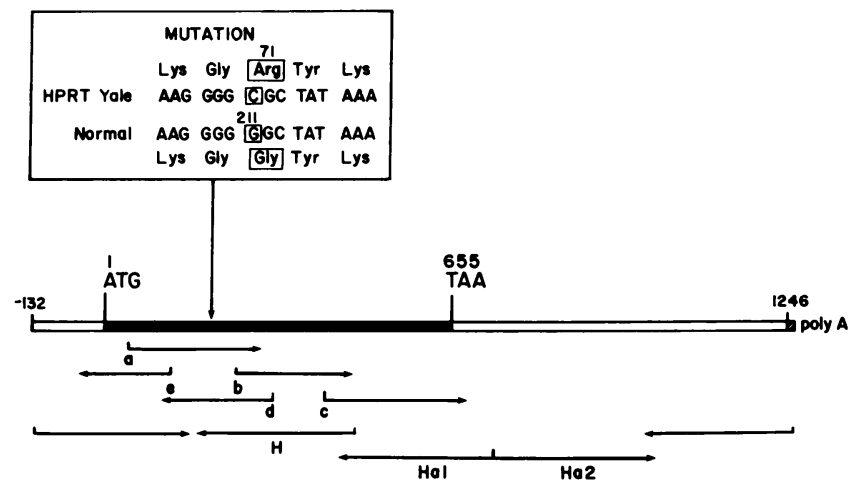

Figure 2. Schematic representation of HPRT $\mathrm{T}_{\text {Yale }} \mathrm{cDNA}\left(\mathrm{pHKT}_{1}\right)$ with position of mutation and sequencing strategy. The coding region is shown in black. The arrows indicate directions and the ranges of sequence determination. The letters indicate locations of HPRTspecific synthetic oligonucleotide primers with sequences represented in $5^{\prime}-3^{\prime}$ orientation: $a$, AGTGATGATGAACCAGG; $b$, CACTGAATAGAAATAGT; $c$, GATATAATTGACACTGG; $d$, CCCCTGTTGACTGGTCA; $e$, AGTCCTGTCCATAATTA. Hal, $\mathrm{Ha} 2$, and $\mathrm{H}$ are digestion fragments of the cDNA with Hae III or Hind III. 


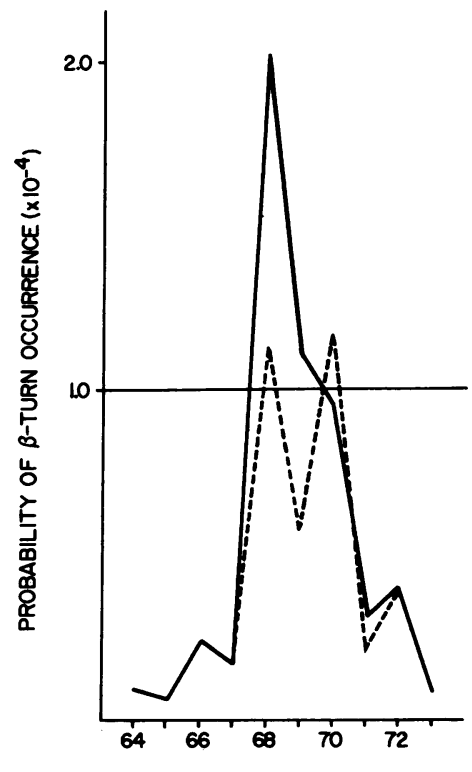

AMINO ACID SEQUENCE NUMBER
Figure 3. Probability of $\beta$ turn occurrence around the region containing the mutation in HPRT Yale. The probability value of a tetrapeptide composed of $i$ to $i$ +3 is plotted against $i$, where $i$ represents the amino acid sequence number with the initial methionine labeled 1 . The solid and broken lines indicate probability of $\beta$-turn occurrence of normal and mutant HPRT, respectively. The horizontal line corresponds to a cut-off value of $1.0 \times 10^{-4}$.

The amino acid substitution predicted in HPRT Yale $_{\text {is lo- }}$ cated within a predicted $\beta$-turn region (Fig. 3). According to the method of Chou and Fasman (16), replacement of glycine with arginine at residue 71 reduces the probability of $\beta$-turn occurrence at residues $68-72$, and elevates its probability at residues 70-73. However, the Pt value at $68-71$ in HPRT $_{\text {Yale }}$ was still greater than the arbitrary cut-off value for $\beta$-turn formation. Furthermore, the increment of the pt value at 70-73

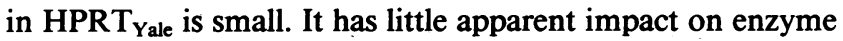
protein stability since the intracellular concentration of HPRT $_{\text {Yale }}$ is normal. Other secondary structural features such as $\alpha$-helix or $\beta$-sheet around the mutation site do not appear to be substantially changed by the glycine to arginine substitution. It is not entirely clear if a change of probabilities of $\beta$-turn occurrence around the mutation site is sufficient to explain the catalytic dysfunction of HPRT Yale $_{\text {. }}$

The substitution of arginine for glycine introduces two additional structural changes. First, arginine has a relatively bulky side chain compared with glycine. Second, a net charge difference occurs. The previously described electrophoretic alteration in HPRT Yale $_{\text {, }}$ cathodal migration during native PAGE (5), is consistent with the substitution of a neutral amino acid (glycine) by a basic amino acid (arginine). Both of these effects alter the regional microenvironment of the protein and probably disrupt normal protein folding. Therefore, this impairment of tertiary structure, as well as the change in net charge, might well affect substrate binding and catalytic activity. This supposition is difficult to evaluate directly due to the absence of residual catalytic activity. Since the three-dimensional tertiary structure of normal human HPRT is not known, the import of structural changes resulting from amino acid substitutions cannot at present be assessed beyond predictions of this sort.

Since most HPRT-deficient subjects have normal levels of mRNA, mutant cDNA cloning is a useful way to identify specific mutations. The marked molecular heterogeneity of human HPRT-deficient states (4-6) thus offers an ideal system for the examination of altered structure-function relationships as well as mutational mechanisms.

\section{Acknowledgments}

The authors thank Dr. C. Thomas Caskey of Baylor University, Houston, TX for his generous gift of human HPRT cDNA. The authors express their gratitude to Mohammad Pashmforoush for RNase mapping analysis of HPRT $_{\text {Yale }}$ mRNA and to Ardith Listeman for her expert secretarial assistance.

This work was supported by National Institutes of Health grant DK-19045-13. Dr. Palella is the recipient of an Arthritis Investigator Award from the Arthritis Foundation.

\section{References}

1. Seegmiller, J. E., F. M. Rosenbloom, and W. N. Kelley. 1967. Enzyme defect associated with a sex-linked human neurological disorder and excessive purine synthesis. Science (Wash. DC). 155:16821684.

2. Kelley, W. N., F. M. Rosenbloom, J. F. Henderson, and J. E. Seegmiller. 1967. A specific enzyme defect in gout associated with overproduction of uric acid. Proc. Natl. Acad. Sci. USA. 57:17351739.

3. Wilson, J. M., A. B. Young, and W. N. Kelley. 1983. Hypoxanthine-guanine phosphoribosyltransferase deficiency. N. Engl. J. Med. 309:900-910.

4. Yang, T. P., P. I. Patel, A. C. Chinault, J. T. Stout, L. G. Jackson, B. M. Hildebrand, and C. T. Caskey. 1984. Molecular evidence for new mutation at the HPRT locus in Lesch-Nyhan patients. Nature (Lond.). 310:412-414.

5. Wilson, J. M., J. T. Stout, T. D. Palella, B. L. Davidson, W. N. Kelley, and C. T. Caskey. 1986. A molecular survey of hypoxanthineguanine phosphoribosyl-transferase deficiency in man. J. Clin. Invest. 77:188-195.

6. Gibbs, R. A., and C. T. Caskey. 1987. Identification and localization of mutations at the Lesch-Nyhan locus by ribonuclease A cleavage. Science (Wash. DC). 236:303-305.

7. Caskey, C. T. 1987. Disease diagnosis by recombinant DNA methods. Science (Wash. DC). 236:1223-1228.

8. Fujimori, S., Y. Hidaka, B. L. Davidson, T. D. Palella, and W. N. Kelley. 1988. Identification of a single nucleotide change in a mutant HPRT gene (HPRT Ann Arbor). Hum. Genet. 79:39-43.

9. Brennand, J., D. S. Konecki, and C. T. Caskey. 1983. Expression of human and Chinese hamster hypoxanthine-guanine phosphoribosyltransferase cDNA recombinants in cultured Lesch-Nyhan and Chinese hamster fibroblasts. Proc. Natl. Acad. Sci. USA. 258:95939596.

10. Maniatis, T., E. F. Fritsch, and J. Sambrook. 1982. Molecular Cloning: A Laboratory Manual. Cold Spring Harbor Laboratory, Cold Spring Harbor, New York. 196-199.

11. Aviv, H., and P. Leder. 1972. Purification of biologically active globin mRNA by chromatography on oligothymidylic acid cellulose. Proc. Natl. Acad. Sci. USA. 69:1408-1412.

12. Polites, H. G., and K. R. Marotti. 1986. A step-wise protocol for cDNA synthesis. Biotechniques. 4:514-520.

13. Benton, W. D., and R. W. Davis. 1977. Screening of $\lambda$ gt recombinant clones by hybridization to a single plaque in situ. Science (Wash. DC). 196:180-182.

14. Sanger, F., S. Nicklin, and A. R. Coulson. 1977. DNA sequencing with chain-terminating inhibitors. Proc. Natl. Acad. Sci. USA. 74:5463-5467.

15. Biggin, M. D., T. J. Gibson, and G. F. Hong. 1983. Buffer gradient gels and ${ }^{35} \mathrm{~S}$ label as an aid to rapid DNA sequence determinations. Proc. Natl. Acad. Sci. USA. 80:3963-3965.

16. Chou, P. Y., and G. D. Fasman. 1978. Empirical predictions of protein conformation. Annu. Rev. Biochem. 47:251-276.

17. Murakami, M. 1985. Mutations affecting the 12th and $61 \mathrm{st}$ amino acids of $\mathrm{p} 21$ protein result in decreased probability of $\beta$-turn occurrence around the mutation positions: a prediction. J. Theor. Biol. 114:193-198. 